\section{ONOMÁVEIN}

Revista semestral de lingüística, filología y traducción
PONTIFICIA UNIVERSIDAD CATÓLICA DE CHILE CATOLICA DE CHILE
FACULTAD DE LETRAS

\title{
Descripción funcional de introducciones de tesis doctorales en las disciplinas de química y lingüística
}

Functional description of doctoral theses introductions in the disciplines of chemistry and linguistics

\author{
Iván Jara Solar* \\ Pontificia Universidad Católica \\ de Valparaíso \\ Chile
}

* Becario Proyecto Mecesup UCV/0311, Programa de Doctorado en Lingüística. Pontificia Universidad Católica de Valparaíso.

ONOMÁZEIN 28 (diciembre de 2013): 72-87

DOI: 10.7764/onomazein.28.7

\section{(C) (i) $\odot$}

Iván Jara Solar: Departamento de Lingüística, Instituto de Literatura y Ciencias del Lenguaje, Pontificia Universidad Católica de Valparaíso. Chile. Correo electrónico: ijara@yahoo.com 


\section{Resumen}

El presente artículo busca describir, mediante los lineamientos del análisis de género, las introducciones de 34 tesis doctorales correspondientes a las disciplinas de química y lingüística presentadas en el período académico 2003-2007 en la Universidad Católica de Valparaíso de Chile. De acuerdo a este análisis, y a partir de la configuración de una propuesta modélica para introducciones de tesis doctorales, el propósito comunicativo de la introducción en una tesis doctoral es justificar la investigación. Destaca la realización de "pasos retóricos" no descritos en la literatura, como definiciones, procedimiento o impacto, así como la ocurrencia optativa de otros como preguntas, procedimiento u originalidad. Otro hallazgo relevante del estudio en la configuración retórica de la introducción es la distinción de dos audiencias, que se han denominado audiencia primaria y audiencia secundaria, referidas a dos diferentes niveles de lectores objetivo.

Palabras clave: introducciones; tesis doctorales; propósitos comunicativos; comunidades disciplinares.

\section{Abstract}

The purpose of this article is to describe, through genre analysis guidelines, introductions of 34 chemistry and linguistics doctoral dissertations. These dissertations were submitted during 2003-2007 academic period to the Universidad Católica de Valparaiso, in Chile. According to this analysis, and from the exemplary configuration of a proposal for doctoral theses introductions, the communicative purpose of introducing a doctoral thesis research is to jus- tify the investigation. A finding that stands out is the use of "rhetorical moves" not described in the literature, such as "definitions", "procedure" or "impact", as well as optional ones like "questions", "procedure" or "originality". At the rhetorical level, another relevant finding of this study is the distinction of two audiences: the primary audience and the secondary one, which refer to two different levels of targeted readers.

Keywords: introductions; doctoral theses; communicative purposes; disciplinary communities. 


\section{Introducción}

La tesis de grado académico constituye uno de los géneros discursivos más antiguos y extendidos para la creación, ratificación y difusión de conocimiento en diferentes disciplinas en el mundo. Se trata de una investigación original que hace parte de una serie de requisitos formales para que un estudiante se gradúe académicamente u obtenga su título profesional.

Las tesis se presentan y defienden al término de un extenso y exigente proceso de aprendizaje del estudiante (ECO, 1990; Paltridge, 1997, 2002; Parry, 1998; Paltridge y Starfield, 2007; PhiIlips y Pugh, 2005; Murray, 2011), y su grado de originalidad varía de acuerdo a cada ámbito disciplinar (Philips y Pugh, 2005) y al grado académico (Tamola, 2005). Sin embargo, se debe precisar que quienes desarrollan una tesis no son necesariamente miembros consolidados de sus comunidades de discurso, sino aspirantes potenciales o satelitales. Esta situación tendría cierto grado de incidencia en el dispar desempeño en tanto escritores.

Si bien esta brecha de conocimiento entre miembros consolidados y aspirantes es más evidente en nivel de pregrado universitario, hay casos en que estudiantes de postgrado no incorporan las prácticas discursivas de sus disciplinas en sus textos sino solo después de un largo proceso de madurez académica, umbral que no siempre coincide con el período de exposición académica (Hyland, 2000, 2002; Carlino, 2005; Samraj, 2008; Parodi, 2008).

Frente a esta realidad sobre las dificultades de estudiantes de postgrado para escribir tesis como un todo (Aitchison, 2003; Bitchener y Basturkmen, 2006; Colás, Buendía y Hernández, 2011; Cubo de Severino, Puatti y Lacon, 2012), se agrega el hecho de que tanto evaluados como evaluadores reconocen en la introducción uno de los segmentos más difíciles de escribir en dicho género (Dudley-Evans, 1986; Shaw, 1991; Bunton, 2002; Flowerdew, 2002; Swales, 1981, 1990, 2004).
No obstante, su papel es clave también en varios otros géneros académicos (Swales, 1990, 2004; Bhatia, 1993, 2004), pues debiera reflejar un posicionamiento adecuado del investigador/escritor respecto a su trabajo mediante una serie de procedimientos reconocidos por los miembros de su comunidad discursiva (audiencia/lector).

Tras comprobar la escasa atención otorgada a las tesis en comparación a la prolífica literatura en torno a otros géneros, como los artículos de investigación científica (AIC), la presente investigación busca describir retóricamente las introducciones de un grupo de tesis doctorales (TD) en dos disciplinas diferentes, pero en un mismo período académico correspondiente a cinco años consecutivos.

La decisión de estudiar géneros disciplinares se basa en que pueden entregar información relevante para la descripción de determinadas áreas de conocimiento, y además aportan a la comprensión de un conjunto de prácticas genéricas similares dentro de un mismo o en diferentes ámbitos (Bhatia, 2004). Así también, este tipo de análisis contribuye al reconocimiento de estructuras lingüísticas prototípicas de un género discursivo, facilitando con ello tanto la producción como la comprensión de un sinnúmero de textos que circulan en diversos contextos institucionales y sociales.

Promover el desarrollo de investigaciones en géneros disciplinares como la TD podría explicar, desde la empiria, una de las aristas de los problemas asociados tanto a la deserción como a la extensión en los plazos de término de estudios doctorales. Así como podría servir de base para la reflexión de los miembros de las diversas comunidades de discurso académicas sobre el rol y la importancia no solo de las tesis, sino también respecto de los espacios de interacción y retroalimentación de los doctorandos durante todo el proceso de investigación.

A continuación, pasaremos a revisar los antecedentes teóricos que sustentan el presente 
estudio, proporcionando definiciones atingentes con el problema de investigación. Luego se presentarán los objetivos, junto con la metodología y los procedimientos, para finalmente entregar los resultados y las conclusiones de los análisis.

\section{Antecedentes teóricos}

\subsection{Hacia una definición del concepto de género}

El concepto de género es tan antiguo como amplio. Su existencia se relaciona, por una parte, con la tendencia del hombre por categorizar la realidad natural y las creaciones humanas, y, por otra, con la necesidad de establecer instancias de regularidad más o menos estables en diferentes esferas de la vida cotidiana, como el comercio, la salud, la educación, entre otros. Ambas aproximaciones al concepto de género tienen en común el hecho de intentar buscar clasificar producciones humanas, esto es, generadas por el ser humano.

No es de extrañar entonces que, desde Aristóteles hasta nuestros días, el género haya sido foco de interés para diversas disciplinas como el derecho, la sociología y la antropología. No obstante, su mayor arraigo se observa en artes como la literatura, el teatro, la música y el cine, entendido como diferentes niveles de categorización a partir de ciertos patrones de estilo, característicos y regulares.

Al margen de los clásicos escritos de Bajtín (1999), de hace casi un siglo, la atención dada por la lingüística aplicada al género como un fenómeno discursivo es más bien reciente. De hecho, revisando la literatura se puede comprobar que la abundante producción, de preferencia con fines pedagógicos, no tiene más de tres o cuatro décadas. No obstante lo anterior, y al contrario de lo que se pudiera suponer, las definiciones de género han sido tan diversas como, en algunos casos, ambiguas.

En este sentido, el trabajo de Hyon (1996) significó un verdadero aporte en la identificación de diferencias y coincidencias del concepto de género en tres influyentes tradiciones lingüísticas contemporáneas: la Nueva Retórica (NR), la Lingüística Sistémica Funcional (LSF) y el Inglés para Propósitos Específicos —English for Specific Purposes (ESP) - . De hecho, autores de estas tres tradiciones reconocen que el reciente interés transversal de la lingüística aplicada hacia el género radica principalmente en aspectos de la didáctica afines con la enseñanza-aprendizaje de la lengua materna y de segundas lenguas en ámbitos académicos o profesionales (Bhatia, 1993, 2004). Al margen de esta importante semejanza, la principal diferencia de estas tres perspectivas está en el foco de sus análisis, pues mientras la NR se centra en las prácticas sociales, la LSF y el ESP se enfocan en los textos.

Una consecuencia directa de esta confluencia de tradiciones es la coexistencia de múltiples definiciones de género discursivo. Desde el ESP, Hyland (2004) proporciona una concepción que evidencia la relación entre este término y la noción de texto, los que frecuentemente suelen encontrarse usados indistintamente, cuando en la realidad se trata de dos niveles distintos. Para Hyland (2004: 1), "genre is a term for grouping texts together, representing how writers typically use language to respond to recurring situations" (el género es un término para agrupar textos que representan cómo prototípicamente los escritores usan el lenguaje para responder a situaciones recurrentes), es decir, los géneros discursivos no existirían sino solo como abstracción de un conjunto de textos. Estos compartirían regularidades retórico-discursivas y rasgos lingüístico-textuales, debido a necesidades de uso y contextos de producción o circulación comunes. Entonces los textos configuran géneros y no a la inversa.

\subsection{Análisis de género y propósito comunicativo}

Los géneros responden a situaciones comunicativas recurrentes. En palabras de Swales (1990), se trata de una clase de evento comu- 
nicativo con propósitos y metas compartidas al interior de comunidades discursivas, cuyos miembros van creando y etiquetando. De esta definición pueden desprenderse algunos de los aspectos centrales del análisis de género, como el papel activo de los integrantes de las comunidades de discurso en la producción, circulación y comprensión de estos textos, así como el importante rol de los propósitos comunicativos.

De acuerdo a lo anterior, los conceptos clave son precisamente: comunidad discursiva y propósito comunicativo. La noción de comunidad discursiva no tiene un origen claro y definido (Swales, 1990; Burgess, 1997), más bien sería fruto de aportes de diferentes disciplinas a lo largo del siglo XX. Sin embargo, Swales (1990) describe las características que la definen, entre las que destacan un conjunto de objetivos comunes, redes de intercomunicación y retroalimentación entre sus miembros, la existencia y circulación de géneros con un léxico específico, entre otras. De hecho, la mayoría de los estudios de género realizados a partir de ejemplares textuales en los últimos 20 años refieren a esta noción de comunidad discursiva.

Respecto al concepto de propósito comunicativo, en los estudios de género se utiliza con bastante frecuencia. Se observa, sin embargo, una aproximación más bien superficial y a veces confusa. Una de las razones de esta situación pudiera ser la complejidad que implica su descripción y representación, y otra un problema de traducción, pues en inglés - lengua de donde provienen la mayoría de los estudios de género- se usan frecuentemente los términos propósito, función y objetivo indistintamente, mientras que desde una perspectiva lingüística en español se sabe que se trata de conceptos diferentes.

Bhatia (1993, 2004) profundiza las propuestas de Swales (1990), aunque solo para el caso específico del discurso profesional, destacando que la construcción de un género está caracterizada primera y principalmente por el propósi- to comunicativo. Bhatia $(1993,2004)$ incluye una condición inequívoca del propósito: la intención del productor/escritor de producir un texto en circunstancias que no, necesariamente, son las mismas por las cuales un comprendedor/lector recurre a él (Parodi, Venegas, Ibáñez y Gutiérrez, 2008).

En este sentido, son las expectativas de escritores y lectores las que juegan un rol trascendente (Carlino, 2006), por cuanto el propósito comunicativo es el objetivo último para el cual un género discursivo es utilizado en un intercambio comunicativo (Parodi y otros, 2008). Esto implicaría la existencia de propósitos menores previos que servirían al propósito mayor.

Bhatia (1993) sostiene que un género contiene a su vez otros géneros, como el resumen o la introducción, que, aunque muy similares en cuanto a su configuración contextual, difieren mucho en cuanto a sus propósitos comunicativos. En consecuencia, y a pesar de estar asociados a un mismo contexto de investigación, son géneros que muestran una estructura retórica diferente y son, por tanto, distintos (Bhatia, 2004).

A partir de esta reflexión de Bhatia (1993) se hace necesario referirse a otro concepto también bastante utilizado, pero poco desarrollado en este tipo de estudios: la retórica. Quizá esta despreocupación se deba al desprestigio que sufrió como disciplina durante siglos. Según Mortara Garavelli (1991), fue la extrema posición antisofista de Platón (siglo IV a. C.) la responsable del extenso oscurantismo de la retórica, posición que, incrementada con posturas de razonamiento matemático, como la de Descartes en el siglo XVIII, la condenó a la indiferencia. Sin embargo, parte del problema respecto al concepto de retórica radica no solo en su precaria explicación, sino también en sus alcances y niveles de abstracción.

La propuesta que mejor satisface esta demanda proviene de la Nueva Retórica (NR), más precisamente desde el ámbito de la producción 
textual, con el concepto de situación retórica, entendida como aquel aspecto abstracto de la escritura, que se compone del tópico acerca del cual se escribe, la audiencia a la que se dirige el texto escrito y la intención o el propósito del mismo (Benítez, 2000). De hecho, la importancia otorgada a la audiencia y al desarrollo de un dispositivo persuasivo para lograr el propósito comunicativo de un texto es también parte esencial en los estudios de género. Se hace necesario, entonces, remarcar que se trata de dos componentes de un marco de análisis complejo, cuya evidencia es textual y cuya unidad de análisis es de nivel retórico: la movida.

La movida retórica - move - se entiende como "a discoursal or rethorical unit that performs a coherent communicative function in a writen or spoken discourse" (Swales, 2004: 229) (una unidad discursiva o retórica que realiza una función comunicativa coherente en un discurso escrito u oral). Como se dijo, el alcance de la movida es retórico-discursivo, y no textual, es decir, no es una unidad concreta y bien delimitada, como las unidades gramaticales, por ejemplo, sino, por el contrario, abstracta. Respecto a su realización, Kanoksilapatham (2007) establece una relación entre la movida y la evidencia textual, que permite su operativización y sistematización. Para esta autora, "a move thus refers to a section of a text that performs a specific communicative function" (Kanoksilapatham, 2007: 23) (una movida refiere a una sección de un texto que realiza una función comunicativa específica). Entonces, a pesar de ser una unidad abstracta, una movida no puede dejar de tener al menos una huella textual que pueda ser rastreada y evidenciada por un lector crítico o un analista experto.

Si el concepto de movida captura el propósito comunicativo de un segmento textual en un nivel más general, el paso — step- explica más expresamente el medio retórico de la realización de la movida. Una movida puede realizarse mediante un solo paso retórico o por la combinación de varios de estos. Según Kanoksilapatham
(2007: 32), cada movida tiene un propósito local que también contribuye al propósito retórico mayor del texto; una suerte de macropropósito comunicativo (Parodi, 2008) o de macronivel como es descrito por algunos autores (Swales, 1990, 2002, 2004; Dudley-Evans, 1994; Bhatia, 1993, 1997, 2004; Kanoksilapatham, 2007). En ambos casos se trataría del propósito comunicativo del género.

\subsection{Géneros y discursos disciplinares}

Las investigaciones que incluyen la variable disciplinar han permitido un mejor reconocimiento y descripción de las regularidades de aquellos géneros insertos en diversos campos de conocimiento. Si bien Becher (2001) admite la dificultad para definir con precisión el concepto de disciplina, concluye que esta tiene identidades reconocibles y atributos culturales particulares. Entre otras, destacan un conjunto de criterios que estipulan que las disciplinas debieran tener una estructura organizacional, una comunidad internacional, organizaciones profesionales y revistas especializadas, características similares a las propuestas revisadas más arriba de Swales (1990) respecto del concepto de comunidad discursiva. De acuerdo a esta relación, no extraña la cercanía conceptual entre la noción de comunidad discursiva y de disciplina. Sin disciplinas no habría comunidad y sin comunidad no habría disciplinas.

Con el fin de resolver el problema de la escritura en un contexto tan complejo de delimitar como el ámbito académico, autores como Hyland (2000) y Bhatia (2004) han propuesto como variable precisamente las disciplinas, comprendiendo así discursos profesionales, científicos, académicos y de cualquier otro ámbito. El foco para esta categoría amplia e integradora no serían ya las artes, las ciencias, las profesiones o los oficios, sino las disciplinas. Esta noción denominada discursos disciplinares por Hyland (2000), y luego reformulada como géneros disciplinares por Bhatia (2004), se refiere principalmente a la relación entre las culturas de las comunidades 
académicas en diferentes disciplinas y sus prácticas discursivas cotidianas.

Ahora bien, los textos que conforman géneros disciplinares se usan para reflejar el uso disciplinar del lenguaje en contextos que son típicos de una comunidad discursiva disciplinar, que se consolida a través de la interacción de sus miembros en áreas de conocimiento y de práctica comunes.

Cabe destacar la importancia de las introducciones, pues desempeñan un papel fundamental en un sinnúmero de géneros disciplinares, bien presentando y demostrando la pertinencia de una investigación científico-académica (Bunton, 2002), o bien promocionándola mediante estrategias de persuasión (Bhatia, 1993, 2004). Como tal, las introducciones establecen entonces las expectativas del lector, haciendo más fácil la lectura de los textos que introducen (Bunton, 2002) y las promesas del escritor. Debido a este complejo entramado retórico-discursivo-textual, estudiantes y evaluadores reconocen que las secciones de introducción son las más difíciles de escribir en una tesis (Dudley-Evans, 1986; Shaw, 1991; Bunton, 2002; Flowerdew, 2002; Swales, 1981, 1990, 2004).

\subsection{Tesis doctorales como género}

La tesis doctoral ha sido abordada desde dos amplias perspectivas, una prescriptiva y otra descriptiva. La primera es no empírica, tiene planteamientos muy generales orientados principalmente a la convención general y desde una mirada más bien teórica (ECo, 1990; Sierra Bravo, 1996; Moyano, 2000), no explica entonces la flexibilidad y el dinamismo propio de las diferentes variedades disciplinares. La segunda en cambio es empírica y permite reconocer eventuales matices sin perder de vista los patrones genéricos. Este tipo de estudios descriptivos se desarrolla a partir de los pioneros trabajos de Swales en 1981 dentro del marco del análisis de género.

Moyano (2000) reconoce que la finalidad de la tesis es informar y acreditar méritos, y su con- tenido es el resultado de un trabajo de investigación original. En esta misma línea, Sierra Bravo (1996) la define como un informe sobre una investigación científica, con la particularidad de que su finalidad es su presentación en una facultad universitaria. Respecto a la TD existe cierto consenso de que se trata de un trabajo escrito en el que el doctorando describe y explica el contenido y los resultados de una investigación científica original efectuada con la orientación de un director.

Desde esta perspectiva descriptiva Swales (2004) concibe la tesis como un género complejo. Este autor considera a la TD como "a rite of passage into the targeted discourse community, or an exit qualification that enables the holder to leave the university world and enter another one" (Swales, 1990: 187) (un rito de iniciación en la comunidad discursiva, o una salida cualificada que permite a los titulares dejar el mundo académico y acceder a otro). Más allá del foco de esta definición, las palabras de Swales (1990) reflejan la importancia de las TD para el acceso a las comunidades discursivas, sean estas académicas o no.

Las diferencias en las TD emergen también si se consideran otras variables como la lengua o el país. De hecho, la TD recibe diferentes nombres de una lengua a otra, de un país a otro, e incluso dentro de una misma universidad. Hyland (2004: 134) explica que "the terms 'thesis' and 'dissertation' are used differently in different countries and sometimes even in different universities in the same countries" (los términos tesis y disertación son usados indiferenciadamente en diferentes países y a veces incluso en diferentes universidades de un mismo país).

Ahora bien, las TD, al igual que las tesis de licenciatura y de magíster, se constituyen de dos momentos complementarios evaluados con diferentes criterios y parámetros, dependiendo de una serie de variables epistemológico-disciplinares (Johns y Swales, 2002) y culturales (Swales, 2004), esto es, la entrega del texto escrito a un co- 
mité evaluador y su posterior defensa oral frente a este jurado.

\section{El estudio}

La presente investigación busca describir las introducciones de un grupo representativo de tesis doctorales en las disciplinas de química y lingüística, a partir de un corpus textual de 34 ejemplares, 18 de química (TDQ) y 16 de lingüística (TDL), presentadas, defendidas y aprobadas en la Pontificia Universidad Católica de Valparaíso de Chile, durante los años académicos comprendidos entre 2003 y 2007 (Corpus TD-2008). Se trata de una muestra censal que recoge el 100\% de los ejemplares susceptibles de ser analizados, es decir, todas las introducciones de TD de este periodo académico.

La pregunta que motiva el presente estudio es ¿para qué y para quién se escriben las introducciones de TD? La respuesta a este interrogante realiza el objetivo general del estudio, cual es comparar las introducciones de tesis doctorales de química y de lingüística mediante la descripción de sus movidas y pasos retóricos, con el fin de configurar un modelo retórico-discursivo de base, tanto para la escritura como la lectura de introducciones en ambas disciplinas.

Para dar cumplimiento a este objetivo se realizó un estudio exploratorio-descriptivo mediante una metodología cualitativa. Es de carácter exploratorio, pues no existen estudios que hayan abordado introducciones en TD en las disciplinas de química y lingüística. Es de alcance descriptivo, puesto que no busca establecer relaciones entre las variables - solo describirlaspara explicar un resultado u otro. Utiliza una metodología cualitativa, por cuanto se trata de un primer análisis a nivel retórico-discursivo, que podría dar la posibilidad de ser abordado más adelante desde una perspectiva cuantitativa o con metodologías de lingüística de corpus.

Las variables de investigación son dos: a) la introducción de TD: segmento textual inicial en una tesis doctoral, y b) las disciplinas: campos de conocimiento (científico-académico-profesional-téc nico) desarrollados con el fin de resolver problemas prácticos o teóricos.

\subsection{El corpus}

A partir del Corpus TD-2008 se constituyó el corpus de introducciones para nuestro estudio. El primero corresponde a la disciplina de química (I-TDQ) y el segundo a la lingüística (I-TDL). La siguiente tabla (tabla 1) muestra los detalles de este corpus.

A partir de este corpus de introducciones se seleccionó un microcorpus aleatorio de 8 introducciones, 4 de cada disciplina, mediante la aplicación de la herramienta de aleatoriedad del programa Microsoft Excel 2003. Con estos 8 ejemplares, que representan el 19\% del total, se configuró una plantilla preliminar de movidas y

\section{TABLA 1}

Conformación del corpus ITD-2008

\begin{tabular}{l|c|c|c|c|c} 
& $\mathbf{N}^{\circ}$ & $\mathbf{N}^{\circ}$ de palabras & Promedio palabras & $\mathbf{N}^{\circ}$ de páginas & Promedio páginas \\
\hline I-TDQ & 18 & 41.913 & 2.329 & 197 & 11 \\
\hline I-TDL & 16 & 28.472 & 1.780 & 80 & 5 \\
\hline ITD-2008 & 34 & 70.385 & --- & 277 & $\ldots$
\end{tabular}


pasos retóricos, con sus correspondientes descripciones y ejemplos. Esta plantilla fue posteriormente piloteada por tres expertos en análisis de género y más tarde ajustada, configurándose así una propuesta de Modelo para Introducciones de Tesis Doctorales (MITD) - ver tabla 2-, la que, después de un proceso de triangulación con expertos de cada disciplina, se aplicó al total del corpus.

Los tres jueces expertos de cada disciplina que intervinieron en la triangulación son miembros activos y reconocidos en sus respectivas comunidades discursivas académicas, así como experimentados evaluadores y directores de TD en los Programas de Doctorado en Ciencias mención Química y de Doctorado en Lingüística de la Pontificia Universidad Católica de Valparaíso de Chile.

\section{Resultados}

Tras aplicarse el MITD a las 34 introducciones de TD, se pudo comprobar tanto su regularidad como su afinidad con modelos anteriores (Swales, 1981, 1990, 2004; Bunton, 2002; Gil, Soler y Carbonell, 2008, 2011). Lo anterior no es de extrañar, por cuanto se trata de introducciones a investigaciones que buscan dar cuenta de un nuevo conocimiento en un contexto epistemológico compartido por una comunidad discursiva disciplinar. Por tanto, de cualquier forma, un estudio de esta envergadura debiera justificarse, reconociendo o desconociendo experiencias disciplinares previas y en ningún caso partiendo de una tabula rasa.

En la tabla 2 se presenta el Modelo para Introducciones de Tesis Doctorales (MITD) elaborado a partir del análisis ya descrito.

Del modelo destaca la convencionalidad-opcionalidad de ciertos pasos retóricos en ambas disciplinas, pues tanto en las I-TDQ como en las I-TDL se presentan facultativamente pasos que tienen una baja ocurrencia, por lo que fueron considerados como pasos opcionales. El umbral

\section{TABLA 2}

Modelo para Introducciones de Tesis Doctorales (MITD)

\begin{tabular}{l|c} 
PASOS & PASOS OPCIONALES \\
CONVENCIONALES & \\
\hline $\begin{array}{l}\text { Movida 1: } \\
\text { Contextualización }\end{array}$ & \\
\hline Paso 1: Relevancia & \\
\hline Paso 2: Generalizaciones & \\
\hline Paso 3: Antecedentes & \\
\hline Paso 4: Definiciones & \\
\hline Movida 2: & \\
Vacío de conocimiento & \\
\hline Paso 1A: Necesidad & Paso 1C: Preguntas \\
\hline Paso 1B: Vacío & Paso 1D: Tradición \\
\hline Movida 3: & \\
Aporte al conocimiento & \\
\hline Paso 1A: Objetivos & Paso 1B: Anuncio de la tesis \\
\hline Paso 1D: Procedimientos & Paso 1C: Metodología \\
\hline Paso 2C: Impacto & Paso 2A: Resultados \\
\hline & Paso 2B: Originalidad \\
\hline & Paso 3: Estructura \\
\hline
\end{tabular}

de convencionalidad que se determinó para ello fue de $50 \%$, que corresponde a la mitad de las posibilidades (0.5) de aparición de un paso en una introducción.

El detalle de la configuración de los pasos retóricos identificados en las introducciones de cada disciplina se presenta en las siguientes dos tablas (tablas 3 y 4).

Solo 15 de los 17 pasos retóricos inicialmente identificados tuvieron más de una aparición en I-TDQ, razón por la que dos pasos quedaron excluidos de la tabla 2: Ios pasos M3-p3 (estructura) y M3-p4 (agradecimientos). Estos tuvieron un porcentaje de aparición de 5,5\%, es decir, una ocurrencia mínima correspondiente a solo una realización de cada uno de estos pasos en las 18 


\section{TABLA 3}

Pasos convencionales y opcionales en I-TDQ

\begin{tabular}{c|c} 
Pasos convencionales & Pasos opcionales \\
\hline M1-p1 (Relevancia) & M2-p1C (Preguntas) \\
\hline M1-p2 (Generalizaciones) & M2-p1D (Tradición) \\
\hline M1-p3 (Antecedentes) & M3-p1B (Anuncio) \\
\hline M1-p4 (Definiciones) & M3-p1C (Metodología) \\
\hline M2-p1A (Necesidad) & M3-p1D (Procedimiento) \\
\hline M2-p1B (Vacío) & M3-p2A (Resultados) \\
\hline M3-p1A (Objetivos) & M3-p2B (Originalidad) \\
\hline M3-p2C (Impacto) &
\end{tabular}

introducciones de tesis doctorales de química.

Respecto de igual procedimiento, aplicado ahora al corpus de introducciones de lingüística, el resultado es como sigue en la tabla 4.

A diferencia de lo que ocurrió en I-TDQ, en las I-TDL no se eliminó ningún paso retórico de los 17 identificados preliminarmente. Un aspecto destacable es que los pasos convencionales se realizan en las movidas 1 y 2 , mientras que la opcionalidad de pasos se da solo en la movida 3 (M3 aporte al conocimiento).

Los 7 pasos retóricos opcionales del MITD (preguntas, tradición, anuncio de la tesis, metodología, resultados, originalidad, estructura) se realizan entre $\mathrm{M}_{2}$ y $\mathrm{M} 3$, relativas a la presentación de un vacío investigativo teórico, práctico o metodológico (M2), y al compromiso investigativo de ocuparlo mediante la generación de un nuevo conocimiento (M3). El MITD queda constituido entonces de 9 pasos retóricos convencionales, distribuidos en las 3 movidas identificadas.

Un paso retórico que presenta una alta ocurrencia, M1-p4 (definiciones), muestra una diferencia entre ambas disciplinas, así como también en lingüística, pues del 75\% (190 pasos en total) de este paso en este ámbito, un 50\% corresponde a definición de autores y un $25 \%$ a propuestas o posturas del tesista, es decir, no tienen referencia de fuente explícita. En cambio, en I-TDQ, el 100\% de los pasos (126 pasos en total) que aparecen son definiciones sin referencia, cuestión que podría explicarse por el nivel de consolidación de conceptos en química, o por el mayor consenso respecto de la denominación de fenómenos como la especiación .

\section{TABLA 4}

Pasos convencionales y opcionales en I-TDL

\begin{tabular}{c|c|c}
\multicolumn{2}{c|}{ Pasos convencionales } & Pasos opcionales \\
\hline M1-p1 (Relevancia) & M2-p1C (Preguntas) & M3-p1C (Metodología) \\
\hline M1-p2 (Generalizaciones) & M2-p1D (Tradición) & M3-p2A (Resultados) \\
\hline M1-p3 (Antecedentes) & M3-p1A (Objetivos) & M3-p2B (Originalidad) \\
\hline M1-p4 (Definiciones) & M3-p1B (Anuncio) & M3-p4 (Agradecimientos) \\
\hline M2-p1A (Necesidad) & M3-p1D (Procedimiento) & \\
\hline M2-p1B (Vacío) & M3-p2C (Impacto) & \\
\hline & M3-p3 (Estructura) &
\end{tabular}

1 Proceso de identificación y cuantificación de diferentes especies, formas o fases químicas presentes en una matriz ambiental. 
Otro resultado relevante es el alto porcentaje (75\%) de presencia en I-TDL del paso retórico que evidencia los paradigmas en los cuales se sostiene la investigación doctoral (M2-p1D, tradición), que mantiene una tradición teórico-metodológica o asume alguna parte de ella, según describe la tabla que presenta las explicaciones de las rúbricas que configuran el MITD — ver tabla 5-. Este mismo paso en I-TDQ marca solo un $6 \%$, indicador de que en química los compromisos teórico-metodológicos estarían más claros o no requerirían mayor explicitación.

\section{TABLA 5}

Explicación de rúbricas del MITD

\begin{tabular}{|c|c|}
\hline & Descripción \\
\hline Movida 1: Contextualización & $\begin{array}{l}\text { Presenta el marco general de la investigación en relación con el } \\
\text { estado actual del conocimiento disciplinar en el que se enmarca } \\
\text { el estudio. }\end{array}$ \\
\hline Paso 1: Relevancia (Mı-p1) & $\begin{array}{l}\text { Se refiere a la relevancia del tema a investigar para hacer avanzar el conocimien- } \\
\text { to disciplinar. }\end{array}$ \\
\hline Paso 2: Generalizaciones (M1-p2) & Representa el conocimiento actual relacionado con el tema a investigar. \\
\hline Paso 3: Antecedentes (M1-p3) & $\begin{array}{l}\text { Da cuenta de investigaciones previas relacionadas con el tema a investigar en } \\
\text { términos teóricos, prácticos o metodológicos. }\end{array}$ \\
\hline Paso 4: Definiciones (Mz-p4) & Entrega definiciones propias del investigador o extraídas de la literatura. \\
\hline Movida 2: Vacío de conocimiento & $\begin{array}{l}\text { Justifica la necesidad de abordar el problema de investigación considerando } \\
\text { algún aspecto del marco general descrito en } \mathrm{Ml}_{1} \text {. }\end{array}$ \\
\hline Paso IA: Necesidad (M2-p1A) & Señala la necesidad de solucionar un problema teórico o aplicado. \\
\hline Paso 1B: Vacío (M2-p1B) & Presenta una brecha en el problema que se desea investigar. \\
\hline Paso 1C: Preguntas (M2-p1C) & Cuestiona a partir de preguntas de investigación abiertas o cerradas. \\
\hline Paso 1D: Tradición (M2-pıD) & Mantiene una tradición teórico-metodológica o asume alguna parte de ella. \\
\hline Movida 3: Aporte al conocimiento & $\begin{array}{l}\text { Presenta la investigación o parte de ella con el fin de satisfacer la necesidad } \\
\text { presentada en M2. }\end{array}$ \\
\hline Paso IA: Objetivos (M3-p1A) & $\begin{array}{l}\text { Se presenta el o los objetivos necesarios para abordar el problema que será in- } \\
\text { vestigado. }\end{array}$ \\
\hline $\begin{array}{l}\text { Paso 1B: Anuncio de la investigación } \\
\text { (M3-p1B) }\end{array}$ & Se anuncia la investigación o parte de ella. \\
\hline Paso 1C: Metodología (Mз-p1C) & Se señala la metodología de la investigación. \\
\hline Paso 1D: Procedimientos (M3-p1D) & Se describe el procedimiento y/o las herramientas de análisis. \\
\hline Paso 2A: Resultados (M3-p2A) & Presenta parte de los hallazgos de la investigación. \\
\hline Paso 2B: Originalidad (M3-p2B) & Enfatiza la originalidad de la investigación. \\
\hline Paso 2C: Impacto (M3-p2C) & Vislumbra proyecciones y aportes de la investigación. \\
\hline Paso 3: Estructura (M3-p3) & Presenta la estructura general de la tesis. \\
\hline Paso 4: Agradecimientos (M3-p4) & Entrega agradecimientos a otros investigadores, proyectos o instituciones. \\
\hline
\end{tabular}


El MITD, aplicable a las disciplinas de química y lingüística, mantiene la secuencia lógico-racional del clásico modelo CARS -Creating a research space - de Swales (1990), pero con nuevos pasos retóricos identificados, a saber: definiciones, necesidad, procedimientos, impacto, que resultaron ser convencionales en ambas disciplinas.

Aunque en general, de acuerdo a los resultados de la triangulación, el límite entre los dos primeros pasos de la movida I (MI, contextualización) es difuso, se mantuvo la separación entre estos en el MITD, pues se trata de pasos con propósitos diferentes. El primero (M1-p1, relevancia) pretende justificar el interés por un determinado tema, mientras que el segundo (M1-p2, generalizaciones) busca presentar un estado de conocimiento, es decir, se enfoca menos al problema de investigación y más a la descripción de situaciones generales. La diferencia entre ambos pasos retóricos estaría dada, entonces, tanto por el nivel de cristalización del conocimiento en la disciplina como por la cercanía temporal del tema a investigar respecto de la realización de la investigación.

En cuanto a los otros dos pasos de la movida I (MI, contextualización), se aprecia una diferencia al interior de las I-TDL, pues mientras más de la mitad de estas presentan definiciones propias del tesista, el resto lo hace con referencia a algún autor de manera general (M1-p3, antecedentes) o específica (M1-p4, definiciones). En las I-TDQ, en cambio, ninguna definición se presenta con referencia, aunque tampoco parecen ser propuestas personales de los tesistas.

Si se pretende establecer una característica prototípica general de las introducciones en tesis doctorales - al menos en química y lingüística一, esta sería la ciclicidad, entendida como la recurrencia de ciertas movidas retóricas mediante diferentes pasos, incluidos aquellos casos donde la última movida (M3, aporte al conocimiento) no se realiza. De hecho, solo una introducción en cada disciplina despliega la se- cuencia retórica lineal y sin reiteraciones.

En lo relativo a los propósitos comunicativos de las TD, en general, tanto a partir del análisis como a través de los comentarios de los jueces expertos en el proceso de triangulación, se puede sostener que los propósitos comunicativos principales son, sin considerar orden de importancia: presentar la investigación, justificar la investigación y demostrar suficiencia investigativa por parte del investigador. El propósito de presentar se dirigiría a una audiencia general, que incluiría tanto a miembros como no miembros de la comunidad discursiva disciplinar (audiencia secundaria); en cambio, los propósitos de justificar y demostrar estarían orientados a una audiencia restringida, esto es, el comité evaluador (audiencia primaria), que en definitiva es la que califica la tesis.

En su propuesta de clasificación por niveles del discurso especializado Gotti (2003) vislumbra al menos tres niveles diferentes de audiencia, a saber, una conformada por especialistas, otra de no especialistas y una tercera formada por legos. Estas variarían en función de las necesidades propias de cada género. La audiencia secundaria estaría conformada tanto por lectores especialistas de otras disciplinas como por legos, mientras en la audiencia primaria solo habría expertos consolidados de las comunidades discursivas disciplinares. Así, la audiencia primaria estaría en condiciones de reconocer las movidas retóricas y los propósitos comunicativos, mientras que la audiencia secundaria solo tendría algunas intuiciones a partir de ciertas huellas textuales, insuficientes para una configuración retórica que permita realizar una evaluación competente.

Respecto a la relación entre las movidas retóricas descritas y los propósitos comunicativos, podría sostenerse que en las movidas 1 y 2 predominaría el propósito de justificar la investigación y demostrar suficiencia investigativa, mientras que en la movida 3 primaría el propósito de presentar la investigación. En todo caso, consi- 
derando que no todas las introducciones realizaron esta última movida, estamos en condiciones de proponer que el macropropósito comunicativo de las introducciones en tesis doctorales es justificar la investigación.

Los propósitos comunicativos de las introducciones de TD de química y lingüística son comunes en ambas disciplinas, en tanto responderían a la solución de problemas de investigación prácticos o metodológicos, como sucedería en química, o a llenar un vacío teórico, como podría ocurrir en lingüística, por ejemplo. Esto quiere decir que estos propósitos, amplios y coherentes con la naturaleza de las TD, permitirían abordar temáticas que no tengan que ver exclusivamente con problemas aplicados, sino también con cuestiones de orden teórico, tanto en ámbitos de las ciencias naturales como de las ciencias sociales y humanas.

\section{Conclusiones}

Desde la perspectiva disciplinar abordada en esta investigación, el análisis de género no solo sirve para aprovechar el poder de generalización que agrupa a los textos que tienen similitudes en términos de propósito retórico y audiencia, sino además para explorar la manera en que se diferencian de una disciplina a otra. Y por qué no, dentro de una misma disciplina.

El análisis de la estructuración retórico-discursiva del corpus de introducciones estudiada evidenció la ciclicidad de las movidas retóricas del MITD. La reiteración cíclica de las movidas mediante la ocurrencia de diferentes pasos retóricos se realiza indistintamente en las disciplinas de química y lingüística. Lo anterior se corresponde con resultados de estudios anteriores en introducciones de AIC (Swales, 1990, 2004) y de TD (Bunton, 2002) que demostraron diversos grados de ciclicidad de las movidas retóricas.

Este fenómeno podría deberse a la no restricción de espacio que tienen las TD, en comparación a otros géneros como los AIC, o a que el problema de investigación requiera una mayor demanda de argumentos que justifiquen su estudio, o simplemente a la inexperiencia de los investigadores/tesistas en cuanto escritores académicos, como miembros satelitales de sus respectivas comunidades discursivas.

Esta falta de experiencia se podría remediar incentivando la participación colaborativa de jóvenes investigadores (Aitchison, Kamler y Lee, 2011) en proyectos de mediana o gran envergadura a cargo de profesores con experiencia, así como estimulando la práctica habitual en otros textos correspondientes a géneros orales o escritos similares (Shaw, 1991; Aitchison y otros, 2011). Todo con el fin de promover espacios de interacción y retroalimentación efectivos entre los miembros consolidados y los aspirantes de las comunidades académicas.

Respecto de la opcionalidad de las movidas, aunque no todas las I-TDQ presentan la movida 3 (aporte al conocimiento), no estamos en condiciones de asegurar que no cumplen los mismos propósitos comunicativos que las I-TDL, por cuanto la movida I (contextualización) y la movida 2 (vacío de conocimiento) son las imprescindibles en la creación de un espacio de investigación (Swales, 1981, 1990, 2004; Gil y otros, 2008, 2011).

Ahora bien, la no realización del paso de presentación de la estructura en las I-TDQ podría deberse a una cuestión de tradición disciplinar, al tiempo que la razón de la ausencia del paso (M3-p4, agradecimientos) sería que en la organización general de las TDQ existe un segmento textual especial para realizar los agradecimientos, el cual no se realiza en las TDL.

Con todo, se observa que las introducciones de TD relacionan complejas unidades textuales-discursivas y cognitivas con la tradición académica que implica su desarrollo en una comunidad discursiva. Esto representa un desafío tanto para el tesista como para su director. Por ello, no es posible fiarse de una categorización en segmentos textuales para su desarrollo o análisis y, 
además, se debe tener conciencia de la perspectiva retórica, la cual puede dar luces acerca de la configuración textual definitiva. Esta rigurosidad obligaría a desarrollar análisis de textos en su globalidad, permitiendo reconocer coherencia entre segmentos textuales y los propósitos comunicativos de las secuencias retóricas que conforman. Lo anterior incidiría en una mayor atención al qué, cómo y a quién se escribe (problema retórico).

En cuanto a la pregunta que motiva este estudio, ¿para qué y para quién se escriben las introducciones de TD?, queda resuelta como sigue: para justificar la investigación a la audiencia primaria, es decir, a los profesores evaluadores. Esto es coherente con los hallazgos de Paltridge (2002) y Paltridge y Starfield (2007) respecto de la existencia de dos grupos de lectores en la configuración retórica de la audiencia de las tesis.

Esta configuración retórica es consistente con la práctica de algunos programas doctorales de celebrar dos defensas orales, una privada, dirigida a los profesores evaluadores, y luego una pública abierta a toda la comunidad. Es posible sugerir que esta modalidad de defensa privada y defensa pública de tesis satisface el propósito menos explícito, pero más importante, de las introducciones de tesis doctoral, esto es, demostrar suficiencia investigativa por parte del tesista, pues es finalmente el comité evaluador (audiencia primaria) quien sanciona y aprueba la investigación.

Sería aconsejable explorar el género defensa oral de tesis doctoral en aspectos retóricos susceptibles de ser descritos en este tipo de presentaciones, con el fin de contrastar los resultados del presente estudio.

\section{Agradecimientos}

Agradezco a Claudia Araya, Paulina Meza y Paulina Orrego por sus aportes y comentarios.

\section{Bibliografía citada}

Aitchison, Claire, 2003: "Thesis writing circles",
Hong Kong Journal of Applied Linguistics 8 (2), 137-154.

Aitchison, Claire, Barbara Kamler y Alison Lee, 2010: Publishing Pedagogies for the Doctorate and Beyond, London: Routledge.

Bajtín, Mijaíl, 1999: Estética de la creación verbal, Madrid: Siglo XXI.

Becher, Tony, 2001: Tribus y territorios académicos: la indagación intelectual y las culturas de las disciplinas, Barcelona: Gedisa.

Benítez, Ricardo, 2000: "La situación retórica: su importancia en el aprendizaje y en la enseñanza de la producción escrita", Signos 33 (48), 49-67.

BhatıA, Vijay, 1993: Analysing genre: Language use in proffesional settings, London: Longman.

BHatia, Vijay, 1997: "Genre-mixing in academic introductions", English for Specific Purposes 16 (3), 181-195.

Bhatia, Vijay, 2004: Worlds of written discourse, Londres: Continuum.

Bitchener, John y Helen Basturkmen, 2006: "Perceptions of the difficulties of postgraduate $L 2$ thesis students writing the discussion section", Journal of English for Academic Purposes 5, 4-18.

Bunton, David, 2002: "Generic moves in Ph.D. thesis introductions" en John Flowerdew (ed.): Academic discourse, Essex: Pearson Education, 57-75.

BuRgESS, Sally, 1997: Discourse variation across cultures: a genre analytic study of writing in linguistics. Tesis doctoral no publicada, University of Reading (UK).

Carlino, Paula, 2005: “Por qué no se completan las tesis en los posgrados?", Revista Venezolana de Educación 9 (30), 415-420.

Carlino, Paula, 2006: La escritura en la investigación, Buenos Aires: Universidad de San Andrés.

Colás, María Pilar, Leonor Buendía y Fuensanta HeRNÁNDEZ, 2009: Competencias científicas para la rea- 
lización de una tesis doctoral, Barcelona: Da Vinci. Cubo de Severino, Liliana, Hilda Puiatti y Nelsi Lacon, (eds.), 2012: Escribir una tesis. Manual de estrategias de producción, Córdoba: ComunicArte.

Dudley-Evans, Tony, 1986: "Genre analysis: an investigation of the introduction and discussion sections of MSc dissertations" en Malcom Coulthard (ed.): Talking about text, Birmingham: English Language Research, University of Birmingham, 128-145.

Dudley-Evans, Tony, 1994: "Genre analysis: an approach to text analysis for ESP" en Malcom Coulthard (ed.): Advances in written text analysis, London: Routledge, 219-228.

Eco, Umberto, 1990: Cómo se hace una tesis, Buenos Aires: Gedisa.

FLOWERDEW, John, 2002: "Introduction: approaches to the analysis of academic discourse in English" en John Flowerdew (ed.): Academic Discourse, Harlow: Longman, 1-17.

GIL, Luz, Carmen Soler y María Carbonell, 2008: “The move-step structure of the introductory sections of Spanish PhD theses", RESLA 21, 85-106.

GIL, Luz, Carmen Soler y María Carbonell, 2011: "A contrastive study of the rhetorical organisation of English and Spanish PhD thesis introductions", English for Specific Purposes 30, 4-17.

GotTl, Maurizio, 2003: Specialized discourse: linguistic features and changing conventions, Berna: Peter Lang.

Hyland, Ken, 2000: Disciplinary discourses: Social interactions in academic writing, Harlow: Pearson Education.

HyLAND, Ken, 2002: Writing: teaching and researching, Harlow: Pearson Education.

HyLAND, Ken, 2004: Genre and second language writing, Michigan: The University of Michigan Press.

Hyon, Sunny, 1996: "Genre in three traditions: implications for ESL”, TESOL Quarterly 30 (4), 693-722.
Johns, Ann M. y John Swales, 2002: "Literacy and disciplinary practices: opening and closing perspectives", Journal of English for Academic Purposes 1, 13-28.

Kanoksilapatham, Budsaba, 2007: "Introduction to move analysis" en Douglas Biber, Ulla Connor y Thomas Upton (eds.): Discourse on the move. Using corpus analysis to describe discourse structure, Amsterdam: John Benjamins, 23-41.

Mortara Garavelli, Bice, 1991: Manual de retórica, Madrid: Cátedra.

Moyano, Estela, 2000: Comunicar ciencia, Buenos Aires: Universidad Nacional de Lomas de Zamora.

MurRAY, Rowena, 2011: How to write a thesis, third edition, Maidenhead: Open University Press.

Paltridge, Brian, 1997: "Thesis and dissertation writing: Preparing ESL students for research", English for Specific Purposes 16(1), 61-70.

Paltridge, Brian, 2002: "Thesis and dissertation writing: an examination of published advice an actual practices", English for Specific Purposes 21, 125-143.

Paltridge, Brian y Sue Starfield, 2007: Thesis and dissertation writing in a second language: A handbook for supervisors, London: Routledge.

PARODI, Giovanni, 2008: Géneros académicos y géneros profesionales: Accesos discursivos para saber y hacer, Valparaíso: Ediciones Universitarias de Valparaíso.

Parodi, Giovanni, René Venegas, Romualdo Ibañez y Rosa María Gutiérrez, 2008: "Géneros del discurso en el Corpus PUCV-2006: criterios, definiciones y ejemplos" en Giovanni Parod (ed.): Géneros académicos y géneros profesionales: Accesos discursivos para saber y hacer, Valparaíso: Ediciones Universitarias de Valparaíso, 39-73.

PARRY, Sharon, 1998: "Disciplinary discourse in doctoral theses", Higher Education 36, 273-299. 
PhILLIPS, Estelle y Dereck Pugh, 2005: How to get a Ph D: A Handbook for students and their supervisors, Maidenhead Berkshire: Open University Press.

Samra, Betty, 2008: "A discourse analysis of master's theses across disciplines with a focus on introductions", Journal of English for Academic Purposes 7, 55-67.

SHaw, Philip, 1991: "Science research students' composing processes", English for Specific Purposes 10, 189-206.

Sierra Bravo, Restituto, 1996: Tesis doctorales y trabajos de investigación científica, Madrid: Paraninfo.

Swales, John, 1981: "Aspects of article introductions", Aston ESP Research Reports, 1.

SwALES, John, 1990: Genre analysis: English in academic and research settings, Cambridge: University of Cambridge.

SwALES, John, 2002: "Integrated and fragmented worlds: EAP materials and corpus linguistics" en John Flowerdew (ed.): Academic discourse, London: Longman, 150-164.

SwALES, John, 2004: Research genres: explorations and applications, Cambridge: University of Cambridge.

TAmola, Diana, 2005: "La tesina de licenciatura” en Liliana Cubo de Severino (coord.): Los textos de la Ciencia. Principales clases del discurso académico-científico. Córdoba: Comunicarte, 235-265. 